\title{
LOBULAR PLEOMORPHIC CARCINOMA VERSUS INVASING LOBULAR CARCINOMA: CLINICOPATHOLOGICAL CHARACTERISTICS AND PROGNOSTIC EVALUATION
}

Iris Rabinovich', Eliza Maiara Jubainski', Chaimaa Chanem³, Natalia de Souza Costa

${ }^{1}$ Universidade Federal do Paraná - Curitiba (PR), Brazil.

${ }_{2}^{2}$ Faculdades Pequeno Príncipe - Curitiba (PR), Brazil.

Introduction: Pleomorphic lobular carcinoma (PLC) is a rare variant of invasive lobular carcinoma (ILC) that has a higher degree of cell atypia and pleomorphism, and has been associated with factors with a worse prognosis such as larger tumor size, and greater lymph node involvement, however, its real prognostic value is still not well defined. Objectives: To evaluate the clinical-pathological characteristics, overall survival, and disease-free survival in PLC and compare it to ILC. Method: All ILC and PLC cases submitted to surgery at Hospital Nossa Senhora das Graças from January $1^{\text {st }}$, 2008 to January $31^{\text {st }}, 2018$ were analyzed. The main clinical and pathological variables related to prognosis were analyzed, as well as the overall survival and disease-free survival for both groups. Results and Discussion: 77 cases of ILC and 35 of PLC were found. The PLC group presented factors of worse prognosis in relation to the ILC group, such as a higher histological grade $(\mathrm{p}<0.001)$, presence of lymphovascular invasion $(\mathrm{p}<0.001)$, greater axillary lymph node involvement $(\mathrm{p}=0.003)$, and higher Ki-67 rates $(\mathrm{p}<0.001)$. Contrary to what was expected, we did not find a greater expression of HER2 ( $\mathrm{p}=0.095)$ in the PLC, and there was also no significant difference between the groups regarding disease-free survival and overall survival. Conclusions: PLC still cannot be considered as an independent variable with a worse prognosis and more studies are needed to better understand this entity. 